\title{
Correction to: Pyruvate Kinase M2 Increases Angiogenesis, Neurogenesis, and Functional Recovery Mediated by Upregulation of STAT3 and Focal Adhesion Kinase Activities after Ischemic Stroke in Adult Mice
}

\author{
Dongdong Chen ${ }^{1} \cdot$ Ling Wei $^{1} \cdot$ Zhi-Ren Liu ${ }^{2}$. Jenny J. Yang ${ }^{2} \cdot$ Xiaohuan Gu ${ }^{1} \cdot$ Zheng Z. Wei $^{1,3} \cdot$ Li-Ping Liu $^{4}$. \\ Shan Ping $\mathrm{Yu}^{1,3}$
}

Published online: 26 June 2018

(C) The American Society for Experimental NeuroTherapeutics, Inc. 2018

\section{Correction to: Neurotherapeutics}

https://doi.org/10.1007/s13311-018-0635-2

The original version of this article was updated to correct the misspelling of Li-Ping Liu's name.

The online version of the original article can be found at https://oi.org/ 10.1007/s13311-018-0635-2

Shan Ping Yu

spyu@emory.edu

1 Department of Anesthesiology, Emory University School of Medicine, 101 Woodruff Circle, Woodruff Memorial Research, Building, Suite 620B, Atlanta, GA 30322, USA

2 Department of Biology, Georgia StateUniversity, GA30303, Atlanta, USA

3 Center for Visual and Neurocognitive Rehabilitation, Veteran's Affair Medical Center, Atlanta, GA 30033, USA

4 Department of Neurology, Beijing Tiantan Hospital, Capital Medical University, Beijing 100050, China 